\title{
Quantum phase transition from superconducting to insulating-like state in a pressurized cuprate
} superconductor

\author{
Yazhou Zhou 1,7, Jing Guo ${ }^{1,2,7}$, Shu Cai ${ }^{1,7}$, Jinyu Zhao ${ }^{1,3}$, Genda Gu ${ }^{4}$, Chengtian Lin ${ }^{5}$, Hongtao Yan ${ }^{1,3}$, \\ Cheng Huang1,3, Chongli Yang ${ }^{1}$, Sijin Long1,3, Yu Gong, ${ }^{6}$ Yanchun Li ${ }^{6}{ }^{6}$, Xiaodong Li ${ }^{6}{ }^{6}$, Qi Wu', \\ Jiangping Hu $\mathbb{1}^{1,3}$, Xingjiang Zhou $\mathbb{1}^{1,2,3}$, Tao Xiang ${ }^{1,3}$ and Liling Sun $\mathbb{1}^{1,2,3} \times$
}

\begin{abstract}
Copper oxide superconductors continue to fascinate the communities of condensed matter physics and material sciences because they host the highest ambient-pressure superconducting transition temperature and unconventional electronic behaviour that are not fully explained ${ }^{1-3}$. Searching for universal links between the superconducting state and its normal metallic state is believed to be an effective approach to elucidate the underlying mechanism of superconductivity. One of the common expectations for copper oxide superconductors is that a metallic phase will appear after the superconductivity is entirely suppressed by chemical doping ${ }^{4-8}$ or the application of a magnetic field ${ }^{9}$. Here we report the first observation of a quantum phase transition from a superconducting state to an insulating-like state as a function of pressure in $\mathrm{Bi}_{2} \mathrm{Sr}_{2} \mathrm{CaCu}_{2} \mathrm{O}_{8+\delta}$ ( $\mathrm{Bi2212}$ ) superconductors with two $\mathrm{CuO}_{2}$ planes in a unit cell for doping below, at and above a level that achieves the highest transition temperature. We also find the same phenomenon in related compounds with a single $\mathrm{CuO}_{2}$ plane as well as three $\mathrm{CuO}_{2}$ planes in a unit cell. This apparently universal phenomenon poses a challenge for achieving a unified understanding of the mechanism of high-temperature superconductivity.
\end{abstract}

Although a huge body of experimental work has been reported for copper oxide (cuprate) superconductors since they were discovered more than thirty years ago ${ }^{10,11}$, the correlation between the superconducting state and its normal state or the neighbouring ground state is still widely debated ${ }^{2,6,12-14}$. By changing the chemical makeup of interleaved charge-reservoir layers, electrons can be added to or removed from the $\mathrm{CuO}_{2}$ planes, resulting in the suppression of the antiferromagnetic insulating state of the parent compound ${ }^{2}$. As the doping level reaches a critical value, superconductivity appears and its transition temperature $\left(T_{c}\right)$ grows to a maximum on doping to an optimal one, then declines for higher doping and finally vanishes at the maximum doping level ${ }^{2,4,7,9,15}$. It is important to recognize that once the superconducting state is completely suppressed by chemical doping, the material undergoes a quantum phase transition from a superconducting state to a metallic state $e^{16-18}$. However, detailed experimental studies of the breakdown of the superconducting state in cuprates are still lacking, and they may be crucial for understanding how the superconducting state melts into or emerges from its neighbouring ground states.

Pressure is an alternative method of tuning the superconductivity beyond the chemical doping or external magnetic field, and it can provide significant information on the evolution of superconductivity, electronic state and crystal structure without changing the chemical composition. On the other hand, it can also provide valuable assistance in the search for superconductors with higher values of $T_{\mathrm{c}}$ at ambient pressure by the substitution of smaller ions ${ }^{19}$. A notably successful application of this strategy leads to the discoveries of important cuprate- and Fe-based superconductors ${ }^{10,20,21}$. Therefore, high-pressure studies on superconductivity can not only help the search for new superconductors but also provide a deeper understanding of the correlation between the superconducting state and its neighbouring normal or ground state ${ }^{22-26}$. To reveal how the superconducting state or non-superconducting state develops-a central issue for understanding the high- $T_{\text {c }}$ superconductivity in cuprates, we performed a series of high-pressure investigations by employing our newly developed state-of-the-art technique-combined in situ high-pressure measurements of the resistance and alternating current (a.c.) susceptibility for the same sample at the same pressure. We studied samples that have been investigated broadly by a variety of methods $\mathrm{s}^{24,27-31}$, namely, the under-doped (UD), optimally doped (OP) and over-doped (OD) $\mathrm{Bi}_{2} \mathrm{Sr}_{2} \mathrm{CaCu}_{2} \mathrm{O}_{8+\delta}$ (Bi2212) superconductors with two $\mathrm{CuO}_{2}$ planes in a unit cell.

Figure 1 shows the results of temperature versus in-plane resistance at different pressures for the UD sample with $T_{c}=74 \mathrm{~K}$ (Fig. 1a,d), the OP sample with $T_{\mathrm{c}}=91 \mathrm{~K}$ (Fig. 1b,e) and the OD sample with $T_{\mathrm{c}}=82 \mathrm{~K}$ (Fig. 1c,f). It is found that the onset $T_{\mathrm{c}}$ of these samples exhibits the same high-pressure behaviour: a slight increase initially and then a monotonous decrease on elevating pressure until it is not detectable. Subsequently, an unexpected insulating-like state appears at pressure $\left({ }_{\mathrm{Pi}}\right)$ of $34.3 \mathrm{GPa}$ for the UD sample, $39.9 \mathrm{GPa}$ for the OP sample and $42.2 \mathrm{GPa}$ for the OD sample. Further, the insulating-like behaviour appears when the pressure is higher than ${ }_{\mathrm{Pi}}$ (Fig. 1a-f), which is a grand surprise because one naively expects that by increasing pressure, the bandwidth should widen and the system should become more metallic. We repeated the measurements on new samples and proved that the results are reproducible

'Institute of Physics, National Laboratory for Condensed Matter Physics, Chinese Academy of Sciences, Beijing, China. ${ }^{2}$ Songshan Lake Materials Laboratory, Dongguan, China. ${ }^{3}$ University of Chinese Academy of Sciences, Department of Physics, Beijing, China. ${ }^{4}$ Condensed Matter Physics \& Materials Science Department, Brookhaven National Laboratory, Upton, NY, USA. ${ }^{5}$ Max-Planck-Institut für Festkörperforschung, Stuttgart, Germany. ${ }^{6}$ nnstitute of High Energy Physics, Chinese Academy of Sciences, Beijing, China. ${ }^{7}$ These authors contributed equally: Yazhou Zhou, Jing Guo, Shu Cai.

凶e-mail: Ilsun@iphy.ac.cn 

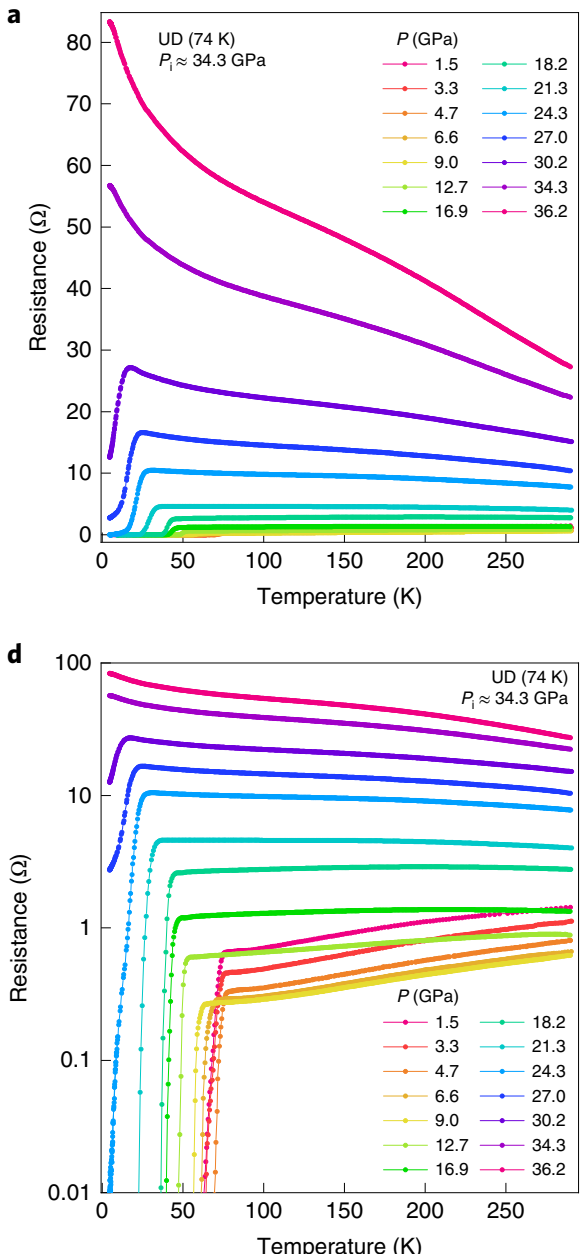
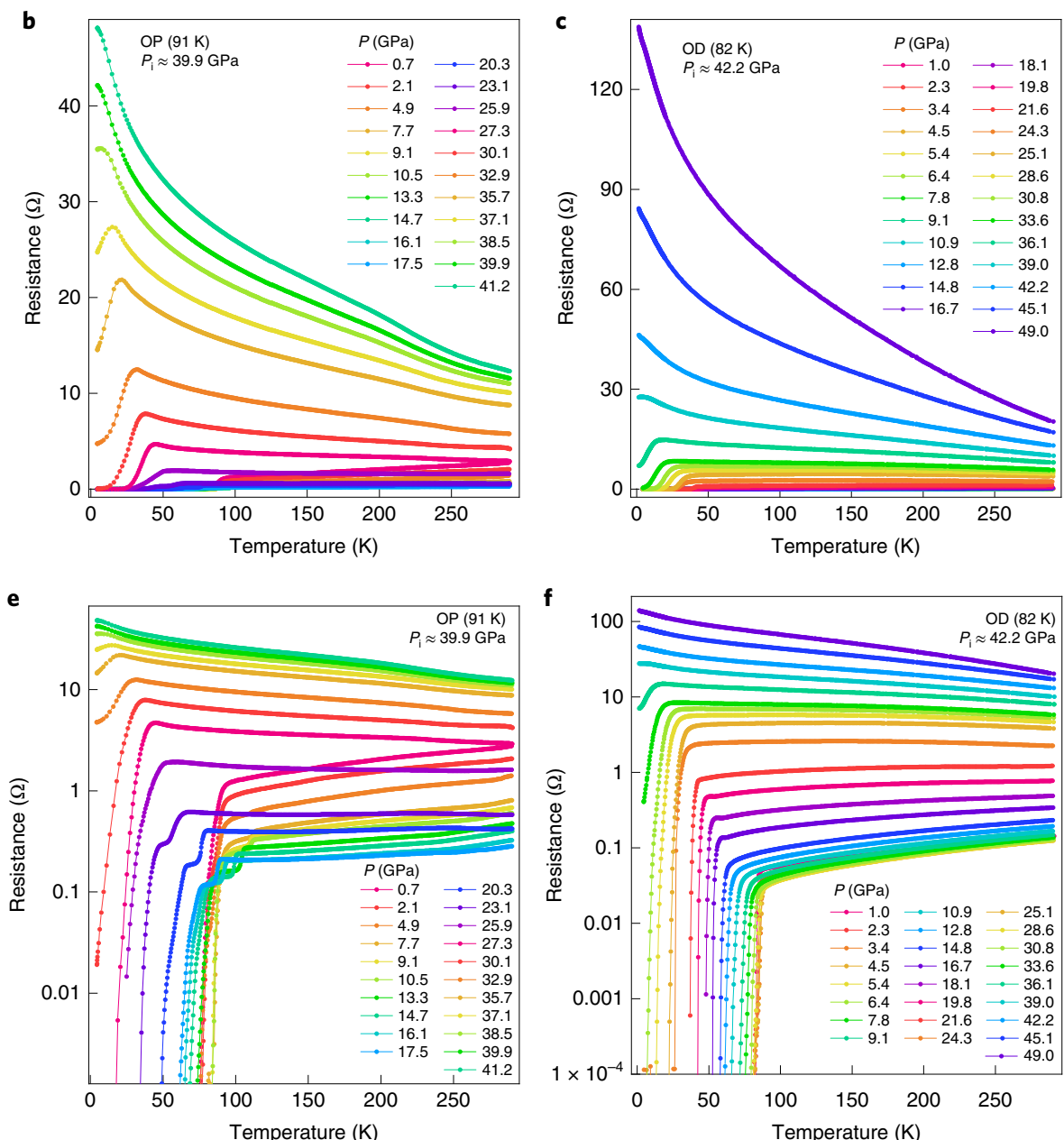

Fig. 1 | Temperature dependence of in-plane resistance for $\mathrm{Bi}_{2} \mathrm{Sr}_{2} \mathrm{CaCu}_{2} \mathrm{O}_{8+\delta}(\mathrm{Bi2212})$ at different pressures. a,d, Plots of temperature versus resistance with linear (a) and $\log (\mathbf{d})$ scales for the UD superconductor in the pressure range of 1.5-36.2 GPa. b,e, OP sample with linear (b) and log (e) scales in the pressure range of 0.7-41.2 GPa; the two-step superconducting transition demonstrates a crossover from two-dimensional to three-dimensional superconducting phases in the pressure range of $4.9-23.1 \mathrm{GPa}$, which is in good agreement with previous studies ${ }^{26}$. $\mathbf{c}, \mathbf{f}$, OD sample with linear (c) and log (f) scales in the pressure range of 1.0-49.0 GPa. The three kinds of samples display the same behaviour of an insulating-like state above pressure ${ }_{\mathrm{Pi}}$.

(Supplementary Fig. 2). Moreover, we found that the transition from the superconducting state to an insulating-like state is reversible when the pressure is released. As shown in Supplementary Fig. 5 , the superconductivity with a zero-resistance state reappears in the sample released from the insulating-like state and a metallic normal state appears. In addition, our careful inspection on the sample recovered from $41.6 \mathrm{GPa}$, by a scanning electronic microscope, demonstrates that no crack is found (Supplementary Fig. 4). These results not only exclude the possibility that the insulating-like state is associated with some pressure-induced cracks but also provides a reasonable explanation on the reversible superconducting transition with a zero-resistance state.

The combined high-pressure measurements of a.c. susceptibility and in-plane resistance were performed for the three kinds of samples mentioned above. The superconducting transitions of the samples detected by a.c. susceptibility can be clearly identified by the onset signal of the deviation from the almost-constant background on the high-temperature side (Fig. 2, blue plots) and the plunge of resistance to zero (Fig. 2, red plots). On compression to $34.3 \mathrm{GPa}$ for the UD sample, $39.9 \mathrm{GPa}$ for the OP sample and $42.2 \mathrm{GPa}$ for the OD sample, all their resistances show an insulating-like behaviour (Fig. 2d,h,l, red plots) and also no diamagnetic signal is captured by the a.c. susceptibility measurements (Fig. 2d,h,l, blue plots). These results reveal that the pressure induces a quantum phase transition from a superconducting state to an insulating-like state in all these superconductors.

We summarize the experimental results in the normalized pressure- $T_{\mathrm{c}}$ phase diagram (Fig. 3, left), which is established on the basis of the pressure- $T_{\mathrm{c}}$ phase diagrams of the UD, OP and OD Bi2212 samples (Fig. 3, right). The phase diagram for the three kinds of samples shows two distinct regions: the superconducting state (SC) and the insulating-like state (I), and demonstrates a universal quantum phase transition from the superconducting to the insulating-like states. It is shown that $T_{\mathrm{c}}$ displays a slight increase initially within a small pressure range, and then a continuous decrease with elevating pressure, in good agreement with the results reported previously ${ }^{32}$. At a critical pressure $P_{c}$ and above (determination of $P_{c}$ is shown in Fig. 3, inset), an insulating-like state emerges (Fig. 3, left) (details of the normalizing analysis are provided in Supplementary Fig. 11). It is worth noting that $P_{\mathrm{c}}$ for the superconductor-insulator transition increases with the increase in the doping level but does not follow the change in $T_{\mathrm{c}}$ (Fig. 3, top). These results demonstrate that the factors determining thermal stability $\left(T_{c}\right)$ and quantum stability $\left(P_{c}\right)$ of the superconducting ground state are not the same. These results 

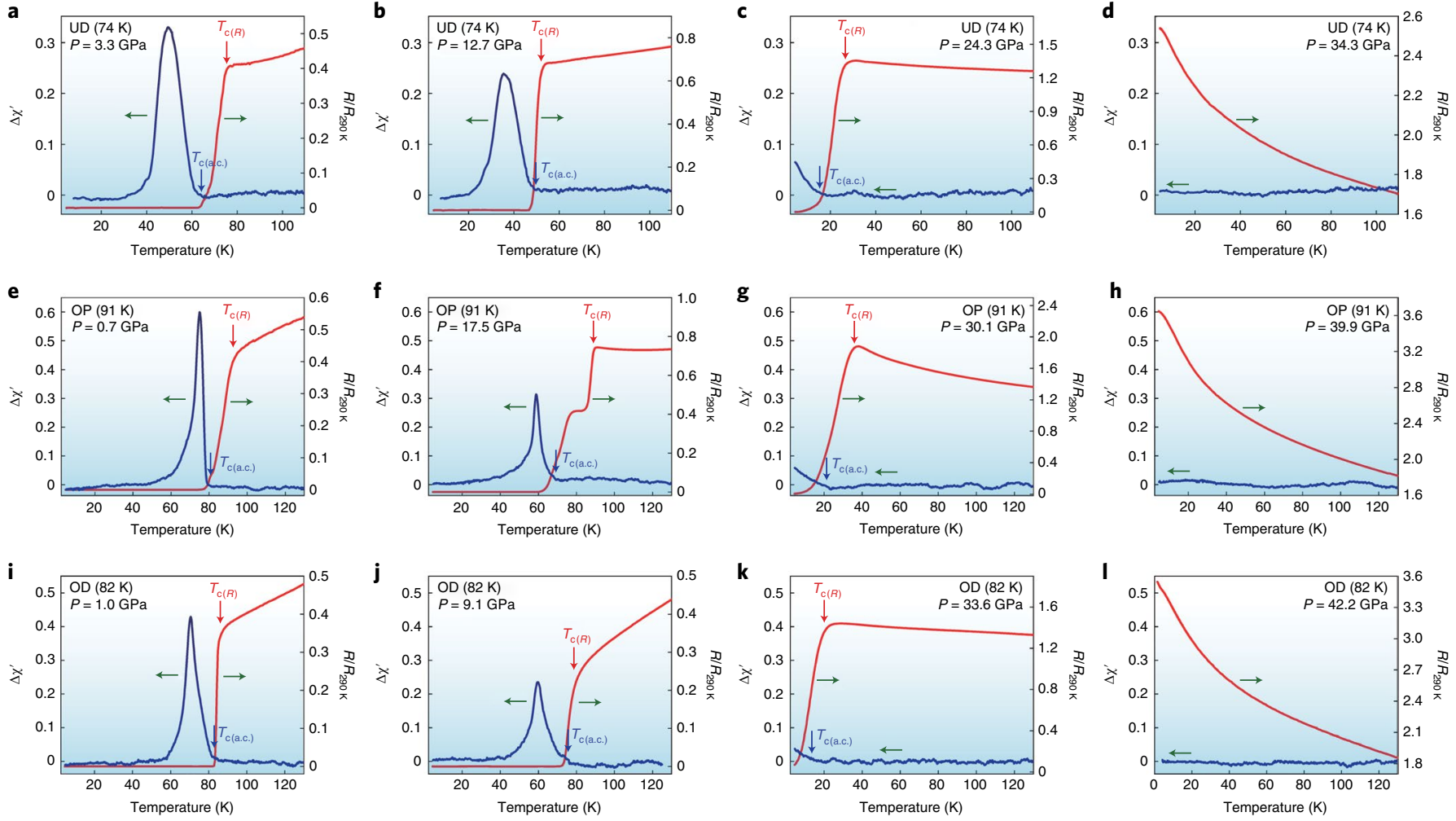

Fig. 2 | In-plane resistance $(\boldsymbol{R})$ and a.c. susceptibility $\left(\Delta \chi^{\prime}\right)$ as a function of temperature (T). a-d, Plots of the UD superconductor. e-h, Plots of the OP superconductor. i-I, Plots of the OD superconductor. The blue lines in the figures are the data for $\Delta \chi^{\prime}(T)$, whereas the red lines are the data for $R(T)$. The red and blue arrows indicate the temperatures of the onset of superconducting transition detected by the resistance ( $\left.T_{c(R)}\right)$ and a.c. susceptibility ( $\left.T_{c(\text { a.c.) }}\right)$ measurements, respectively.
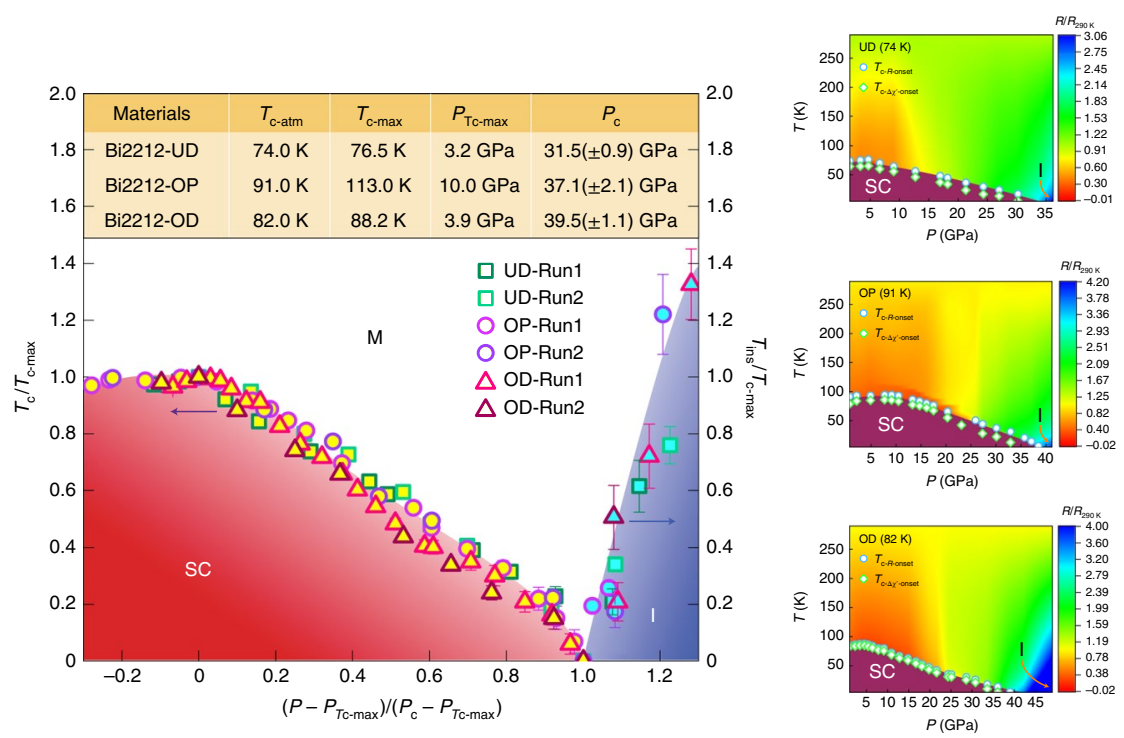

Fig. $3 \mid$ Pressure- $T_{c}$ phase diagrams for $\mathrm{Bi}_{2} \mathrm{Sr}_{2} \mathrm{CaCu}_{2} \mathrm{O}_{8+\delta}$ superconductors. Left, a normalized phase diagram that is built on the basis of the experimental phase diagrams (right). Right: phase diagrams established by the experimental results from the UD, OP and OD samples, together with the mapping information of temperature- and pressure-dependent $R$ (shown in the colour scale). $P_{\mathrm{T}-\mathrm{max}}$ and $P_{\mathrm{c}}$ stand for the pressures where $T_{\mathrm{c}}$ reaches the maximum and zero, respectively. $T_{c-a t m}$ stands for the superconducting transition temperature at ambient pressure. In the normalizing analysis, we define the pressure as $P_{T-\text { max }}$ when $\left(P-P_{T-\text { max }}\right) /\left(P_{c}-P_{T-\text { max }}\right)=0$ and define the pressure as $P_{c}$ when $\left(P-P_{T-\text { max }}\right) /\left(P_{c}-P_{T-\text { max }}\right)=1$. The results of the normalizing analysis for $T_{c} / T_{c-\text { max }}$ versus $\left(P-P_{\pi-\text { max }}\right) /\left(P_{c}-P_{\pi-\text { max }}\right)$ and $T_{\text {ins }} / T_{c-\text { max }}$ versus $\left(P-P_{t c-\text { max }}\right) /\left(P_{c}-P_{\pi-\text { max }}\right)$ show that the three kinds of samples display a universal quantum phase transition from the superconducting state to an insulating-like state. SC, M and I stand for superconducting state, metallic state and insulating-like state, respectively. The region of the $\mathrm{M}$ phase is determined by the critical value of $R / R_{290 \mathrm{~K}}$, where the quantum phase transition occurs. $T_{c-R-\text { onset }}$ and $T_{c-\Delta x^{\prime}-\text { onset }}$ denote the onset temperatures of the superconducting transition detected by the resistance and a.c. susceptibility measurements, respectively. $T_{c-m a x}$ and $T_{\text {ins }}$ are the maximum value of $T_{c}$ and the characteristic temperature of the insulating-like transition, respectively. $P_{c}$ represents the critical pressure from the superconducting state to insulating-like state (methods for determining $T_{\text {ins }}$ and $P_{c}$ are shown in Supplementary Fig. 11). The details about determining error bars can be found in SI. 

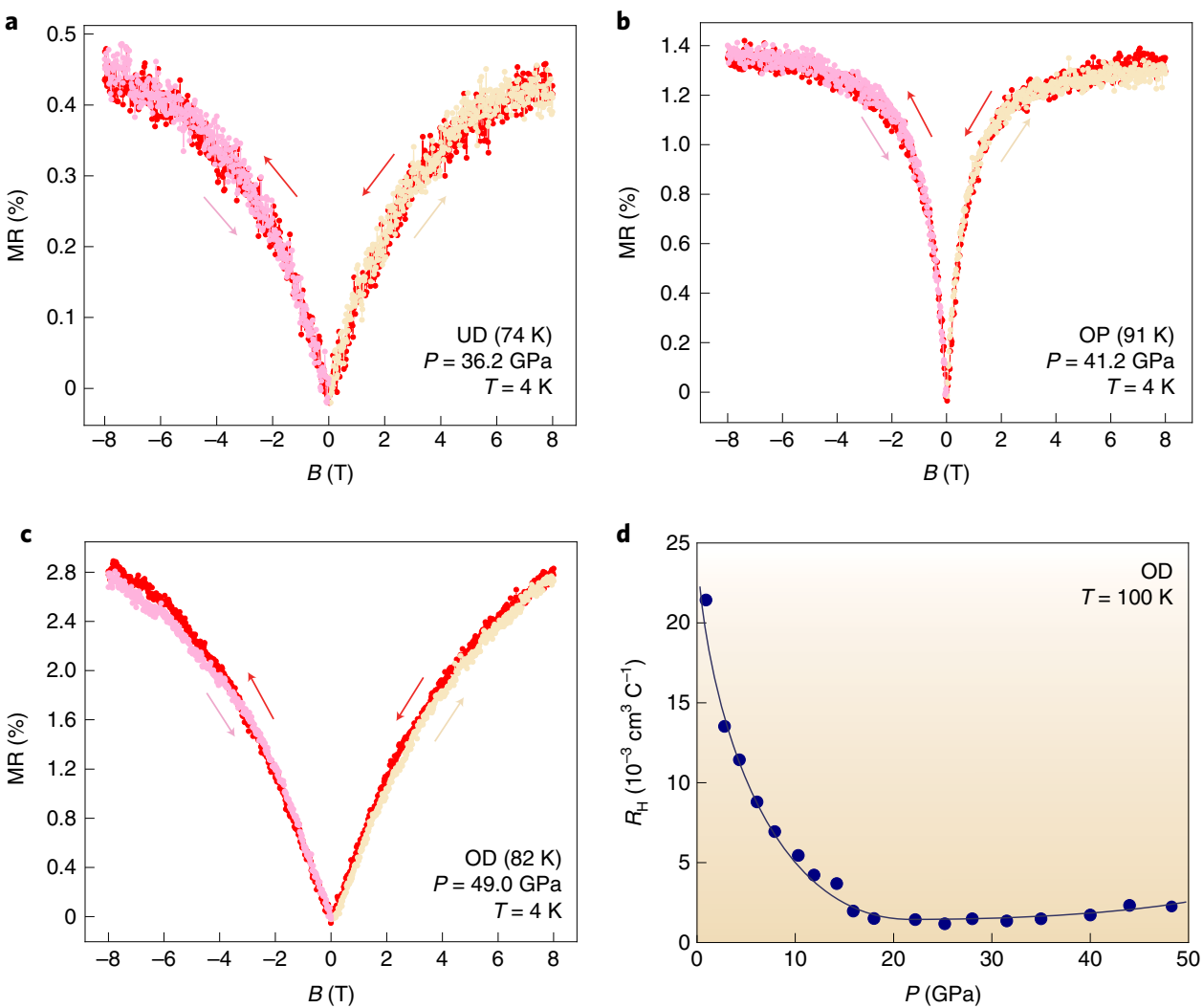

Fig. 4 | Magnetoresistance (MR) of the UD, OP and $\mathrm{OD} \mathrm{Bi}_{2} \mathrm{Sr}_{2} \mathrm{CaCu}_{2} \mathrm{O}_{8+\delta}$ superconductors, and Hall coefficient of the OP sample under pressure. a-c, Plots of MR versus $B$ for the UD (a), OP (b) and OD (c) samples measured at $4 \mathrm{~K}$ and $36.2 \mathrm{GPa}$ for the UD sample, 41.2 GPa for the OP sample and $49 \mathrm{GPa}$ for the OD sample. It is seen that all of them exhibit a positive magnetic effect. The red and beige arrows represent the directions of increasing and decreasing magnetic field, respectively. $\mathbf{d}$, Pressure dependence of the Hall coefficient $\left(R_{\mathrm{H}}\right)$ for the OD superconductor measured at $100 \mathrm{~K}$.

raise two important questions: what is the factor that determines the value of $T_{c}$ and what is the factor that controls the $P_{c}$ value of the superconducting ground state? Further, how do they connect with each other? These questions are still open and call for further investigations from a broader perspective.

To know whether the quantum phase transition discovered in this study is a common phenomenon beyond the Bi2212 superconductors investigated, we conducted the same measurements on the $\mathrm{Bi}_{2} \mathrm{Sr}_{1.63} \mathrm{La}_{0.37} \mathrm{CuO}_{6+\delta}(\mathrm{Bi} 2201)$ superconductor with one $\mathrm{CuO}_{2}$ plane as well as the $\mathrm{Bi}_{2} \mathrm{Sr}_{2} \mathrm{Ca}_{2} \mathrm{Cu}_{3} \mathrm{O}_{10+\delta}(\mathrm{Bi} 2223)$ superconductor with three $\mathrm{CuO}_{2}$ planes in a unit cell. The same phenomenon is also found in these superconductors (Supplementary Fig. 3), indicating that the observed quantum phase transition is universal in bismuth-bearing cuprate superconductors, regardless of the doping level and number of $\mathrm{CuO}_{2}$ planes in a unit cell.

These results impact our knowledge about cuprate superconductors that after the superconducting state is destroyed, the sample should show a metallic state because pressure generally increases the bandwidth. To clarify the possible origin that leads to the destruction of the superconducting state and the emergence of an insulating-like state under pressure, we carried out more experiments.

First, we conducted the high-pressure synchrotron X-ray diffraction measurements at $50 \mathrm{~K}$ for the OD sample on $4 \mathrm{~W} 2$ beamline at the Beijing Synchrotron Radiation Facility. Our results indicated that there is no structural phase transition in the range of pressures up to $43.1 \mathrm{GPa}$, except that the volume of the lattice is apparently compressed (Supplementary Fig. 9). These results ruled out the possibility that the quantum phase transition from superconducting to insulating-like states connects with a pressure-induced structural phase transition.
Second, we measured the magnetoresistance (MR) at $4 \mathrm{~K}$ for the compressed UD, OP and OD samples that host the insulating-like state. The magnetic field was applied perpendicular to the $a-b$ plane of these samples. As shown in Fig. $4 a-c$, the MR values of all the samples exhibit a positive effect. These results are reminiscent of what has been seen in disordered superconducting films. When the film samples move into an insulating state (driven by the magnetic field) from a superconducting state, a positive MR value indicating a superconducting fluctuation state exists on increasing the field ${ }^{33-35}$. These results lead us to consider that the positive MR effect may be a universal feature manifesting the existence of a superconducting fluctuation in the insulating state induced either by a magnetic field in the film samples or pressure in the bulk cuprates. As only a small MR value (of the order of $1 \%$ ) is observed in the insulating-like state of our bulk cuprate superconductor, it implies that the superconducting fluctuation-if it indeed exists in these samples-should be weak.

Third, we performed the high-pressure Hall coefficient $\left(R_{\mathrm{H}}\right)$ measurements for the OD sample (Fig. 4d; measurement details can be found in Supplementary Fig. 1) and find that $R_{\mathrm{H}}(P)$ decreases remarkably with increasing pressure up to approximately $18 \mathrm{GPa}$. Because the Hall resistance versus magnetic field displays a linear behaviour in the pressure range investigated (Supplementary Fig. 6), a typical feature of the single-band system-the decrease in $R_{\mathrm{H}}(P)$ below $18 \mathrm{GPa}$-ought to be associated with the enhancement in carrier density. However, $R_{\mathrm{H}}$ remains almost unchanged for pressures ranging from approximately 18.0 to $35.0 \mathrm{GPa}$ and then shows a slow increase from approximately 35.0 to $48.3 \mathrm{GPa}$. No apparent change in $R_{\mathrm{H}}(P)$ at $P_{\mathrm{c}}=39.5 \mathrm{GPa}$ implies that the total density of charge carriers seems to remain in a steady state with a very slow change when 
crossing quantum criticality. The reproducible result is also obtained in the Bi2201 superconductor (Supplementary Information).

It is noted that unlike the usual insulator, the low-temperature resistance in the insulating-like state rises way too slowly to be exponential. We attempted to fit the low-temperature resistance with exponential dependence and power law, but they failed (Supplementary Fig. 12). Slow rises of this kind have been found in low-temperature orthorhombic $\mathrm{La}_{2-x} \mathrm{Sr}_{x} \mathrm{CuO}_{4}, \mathrm{YiBa}_{2} \mathrm{Cu}_{3} \mathrm{O}_{7-\delta}$ cuprates and $\mathrm{La}_{1-x} M_{x} \mathrm{OBiS}_{2}(M=\mathrm{Ti}$ or Th$)$, which are perceived as fairly mysterious ${ }^{36-38}$.

There is, in fact, no precedence anywhere else for such a transition from a superconducting state to an insulating-like state, to the best of our knowledge. Therefore, some questions are naturally raised: why do itinerant superconducting electrons become localized after the quantum phase transition and what is the exotic pathway that results in the quantum phase transition? All the above are attractive issues in searching for new physics behind the pressure-induced quantum phase transition from a superconducting state to an insulating-like state, which deserves further investigation with other advanced experimental probes and sophisticated theoretical studies.

\section{Online content}

Any methods, additional references, Nature Research reporting summaries, source data, extended data, supplementary information, acknowledgements, peer review information; details of author contributions and competing interests; and statements of data and code availability are available at https://doi.org/10.1038/ s41567-022-01513-2.

Received: 22 May 2021; Accepted: 14 January 2022; Published online: 17 February 2022

\section{References}

1. Chu, C. W., Deng, L. Z. \& Lv, B. Hole-doped cuprate high temperature superconductors. Physica C Supercond. 514, 290-313 (2015).

2. Keimer, B., Kivelson, S. A., Norman, M. R., Uchida, S. \& Zaanen, J. From quantum matter to high-temperature superconductivity in copper oxides. Nature 518, 179-186 (2015).

3. Zaanen, J. Condensed-matter physics: superconducting electrons go missing. Nature 536, 282-283 (2016).

4. Greene, R. L., Mandal, P. R., Poniatowski, N. R. \& Sarkar, T. The strange metal state of the electron-doped cuprates. Annu. Rev. Condens. Matter Phys. 11, 213-229 (2020).

5. Sacépé, B., Feigel'man, M. \& Klapwijk, T. M. Quantum breakdown of superconductivity in low-dimensional materials. Nat. Phys. 16, 734-746 (2020).

6. Božović, I., He, X., Wu, J. \& Bollinger, A. T. Dependence of the critical temperature in overdoped copper oxides on superfluid density. Nature 536, 309-311 (2016).

7. Barisic, N. et al. Universal sheet resistance and revised phase diagram of the cuprate high-temperature superconductors. Proc. Natl Acad. Sci. USA 110 , 12235-12240 (2013).

8. Norman, M. R. \& Pépin, C. The electronic nature of high temperature cuprate superconductors. Rep. Prog. Phys. 66, 1547-1610 (2003).

9. Proust, C. \& Taillefer, L. The remarkable underlying ground states of cuprate superconductors. Annu. Rev. Condens. Matter Phys. 10, 409-429 (2019).

10. Wu et al. Superconductivity at $93 \mathrm{~K}$ in a new mixed-phase $\mathrm{Yb}-\mathrm{Ba}-\mathrm{Cu}-\mathrm{O}$ compound system at ambient pressure. Phys. Rev. Lett. 58, 908-910 (1987).

11. Bednorz, J. G. \& Mller, K. A. Possible high $T_{\mathrm{c}}$ superconductivity in the Ba-La-Cu-O system. Z. Physik B-Condensed Matter 64, 189-193 (1986).

12. Zaanen, J. Planckian dissipation, minimal viscosity and the transport in cuprate strange metals. SciPost Phys. 6, 061 (2019).

13. Zaanen, J. et al. Towards a complete theory of high $T_{\text {c }}$. Nat. Phys. 2, 138-143 (2006).

14. Lee, P. A., Nagaosa, N. \& Wen, X.-G. Doping a Mott insulator: physics of high-temperature superconductivity. Rev. Mod. Phys. 78, 17-85 (2006).

15. Nakamae, $\mathrm{S}$. et al. Electronic ground state of heavily overdoped nonsuperconducting $\mathrm{La}_{2-x} \mathrm{Sr}_{x} \mathrm{CuO}_{4}$. Phys. Rev. B 68, 100502 (2003).
16. Vignolle, B. et al. Quantum oscillations in an overdoped high- $T_{\mathrm{c}}$ superconductor. Nature 455, 952-955 (2008).

17. Platé, M. et al. Fermi surface and quasiparticle excitations of overdoped $\mathrm{Tl}_{2} \mathrm{Ba}_{2} \mathrm{CuO}_{6+\delta}$. Phys. Rev. Lett. 95, 077001 (2005).

18. Hussey, N. E., Abdel-Jawad, M., Carrington, A., Mackenzie, A. P. \& Balicas, L. A coherent three-dimensional Fermi surface in a high-transition-temperature superconductor. Nature 425, 814-817 (2003).

19. Chu, C. W. et al. Evidence for superconductivity above $40 \mathrm{~K}$ in the La-Ba-Cu-O compound system. Phys. Rev. Lett. 58, 405-407 (1987).

20. Kamihara, Y., Watanabe, T., Hirano, M. \& Hosono, H. Iron-based layered superconductor $\mathrm{LaO}_{1-x} \mathrm{~F}_{x} \mathrm{FeAs}(x=0.05-0.12)$ with $T_{c}=26 \mathrm{~K}$. J. Am. Chem. Soc. 130, 3296-3297 (2008).

21. Zhi-An, R. et al. Superconductivity at $55 \mathrm{~K}$ in iron-based F-doped layered quaternary compound $\mathrm{Sm}\left[\mathrm{O}_{1-x} \mathrm{~F}_{x}\right]$ FeAs. Chin. Phys. Lett. 25, 2215-2216 (2008).

22. Deng, L. et al. Higher superconducting transition temperature by breaking the universal pressure relation. Proc. Natl Acad. Sci. USA 116, 2004-2008 (2019).

23. Sun, L. et al. Re-emerging superconductivity at 48 kelvin in iron chalcogenides. Nature 483, 67-69 (2012).

24. Guo, J. et al. Crossover from two-dimensional to three-dimensional superconducting states in bismuth-based cuprate superconductor. Nat. Phys. 16, 295-300 (2020).

25. Takahashi, H. et al. Superconductivity at $43 \mathrm{~K}$ in an iron-based layered compound $\mathrm{LaO}_{1-x} \mathrm{~F}_{x} \mathrm{FeAs}$. Nature 453, 376-378 (2008).

26. Schilling, J. S. What High Pressure Studies Have Taught us About High-Temperature Superconductivity. in Frontiers of High Pressure Research II: Application of High Pressure to Low-Dimensional Novel Electronic Materials 345-360 (Springer, 2001).

27. $\mathrm{Du}, \mathrm{Z}$. et al. Imaging the energy gap modulations of the cuprate pair-density-wave state. Nature 580, 65-70 (2020).

28. Gao, Q. et al. Electronic evolution from the parent Mott insulator to a superconductor in lightly hole-doped $\mathrm{Bi}_{2} \mathrm{Sr}_{2} \mathrm{CaCu}_{2} \mathrm{O}_{8+\delta}$. Chinese Phys. Lett. 37, 087402 (2020)

29. Zhao, H. et al. Charge-stripe crystal phase in an insulating cuprate. Nat. Mater. 18, 103-107 (2019).

30. Kondo, T. et al. Disentangling Cooper-pair formation above the transition temperature from the pseudogap state in the cuprates. Nat. Phys. 7, 21-25 (2011)

31. Parker, C. V. et al. Fluctuating stripes at the onset of the pseudogap in the high- $T_{\mathrm{c}}$ superconductor $\mathrm{Bi}_{2} \mathrm{Sr}_{2} \mathrm{CaCu}_{2} \mathrm{O}_{8+x}$. Nature 468, 677-680 (2010).

32. Adachi, S. et al. Pressure effect in $\mathrm{Bi}-2212$ and $\mathrm{Bi}-2223$ cuprate superconductor. Appl. Phys. Express 12, 043002 (2019).

33. Sambandamurthy, G., Engel, L. W., Johansson, A. \& Shahar, D. Superconductivity-related insulating behavior. Phys. Rev. Lett. 92, 107005 (2004).

34. Breznay, N. P., Steiner, M. A., Kivelson, S. A. \& Kapitulnik, A. Self-duality and a Hall-insulator phase near the superconductor-to-insulator transition in indium-oxide films. Proc. Natl Acad. Sci. USA 113, 280-285 (2016).

35. Mitra, S., Tewari, G. C., Mahalu, D. \& Shahar, D. Negative magnetoresistance in amorphous indium oxide wires. Sci. Rep. 6, 37687 (2016).

36. Adachi, T. et al. Magnetic-field effects on the charge-spin stripe order in La-214 high- $T_{\text {c }}$ cuprates. J. Phys. Conf. Ser. 51, 259-262 (2006).

37. Muramatsu, T., Pham, D. \& Chu, C. W. A possible pressure-induced superconducting-semiconducting transition in nearly optimally doped single crystalline $\mathrm{YBa}_{2} \mathrm{Cu}_{3} \mathrm{O}_{7-\delta}$. Appl. Phys. Lett. 99, 052508 (2011).

38. Fang, Y., Yazici, D., Jeon, I. \& Maple, M. B. High-pressure effects on nonfluorinated $\mathrm{BiS}_{2}$-based superconductors $\mathrm{La}_{1-x} M_{x} \mathrm{OBiS}_{2}(M=\mathrm{Ti}$ and $\mathrm{Th})$. Phys. Rev. B 96, 214505 (2017).

Publisher's note Springer Nature remains neutral with regard to jurisdictional claims in published maps and institutional affiliations.

Open Access This article is licensed under a Creative Commons Attribution 4.0 International License, which permits use, sharing, adap tation, distribution and reproduction in any medium or format, as long as you give appropriate credit to the original author(s) and the source, provide a link to the Creative Commons license, and indicate if changes were made. The images or other third party material in this article are included in the article's Creative Commons license, unless indicated otherwise in a credit line to the material. If material is not included in the article's Creative Commons license and your intended use is not permitted by statutory regulation or exceeds the permitted use, you will need to obtain permission directly from the copyright holder. To view a copy of this license, visit http://creativecommons. org/licenses/by/4.0/.

(c) The Author(s) 2022 


\section{Data Availability}

Source data are provided with this paper. Other data that support the findings of this study are available from the corresponding author upon reasonable request.

\section{Acknowledgements}

We thank J. Zaanen, C.-W. Chu, L. Deng, X. Chen and Y. Zhou for helpful discussions on this work. The work in China was supported by the National Key Research and Development Program of China (grant nos. 2017YFA0302900 and 2017YFA0303103), the NSF of China (grant nos. U2032214, 12122414, 11888101 and 12004419) and the Strategic Priority Research Program (B) of the Chinese Academy of Sciences (grant no. XDB25000000). We appreciate support from the Users with Excellence Program of Hefei Science Center CAS (2020HSC-UE015). Part of the work is supported by the Synergic Extreme Condition User System. J.G. is grateful for support from the Youth Innovation Promotion Association of the CAS (2019008). The work in BNL was supported by the US Department of Energy, Office of Basic Energy Sciences (contract no. desc0012704).

\section{Author contributions}

L.S., T.X. and Q.W. designed the study and supervised the project. G.G. grew the $\mathrm{Bi}_{2} \mathrm{Sr}_{2} \mathrm{CaCu}_{2} \mathrm{O}_{8+\delta}$ single crystals. H.Y. and X.Z. grew the $\mathrm{Bi}_{2} \mathrm{Sr}_{1.63} \mathrm{La}_{0.37} \mathrm{CuO}_{6+\delta}$ single crystals. C.T. L. grew the $\mathrm{Bi}_{2} \mathrm{Sr}_{2} \mathrm{Ca}_{2} \mathrm{Cu}_{3} \mathrm{O}_{10+8}$ single crystals. Y.Z., J.G., S.C., J.Z. and L.S. performed the high-pressure resistance, a.c. susceptibility and magnetroresistance measurements. J.G., Y.Z., Y.G., Y.L., X.L. and C.Y. performed the high-pressure X-ray diffraction measurements. J.G., C.H. and S.L. performed the high-pressure Hall measurements. L.S., T.X., Q.W., J.H. and Y.Z. wrote the manuscript in consultation with all the authors.

\section{Competing interests}

The authors declare no competing interests.

\section{Additional information}

Supplementary information The online version contains supplementary material available at https://doi.org/10.1038/s41567-022-01513-2.

Correspondence and requests for materials should be addressed to Liling Sun.

Peer review information Nature Physics thanks Nandini Trivedi and the other, anonymous, reviewer(s) for their contribution to the peer review of this work.

Reprints and permissions information is available at www.nature.com/reprints. 